Rev. Elev, Méd. vét. Pays trop., 1978, 31 (3) : 363-368.

\title{
Production de protéines d'organismes unicellulaires (P.O. U.) à partir de coque d'arachide
}

\author{
par J. BLANCOU $(*)$, H. CALVET (**) et R. RIVIÈRE (***) \\ (avec la collaboration technique de A. NIANG)
}

\begin{abstract}
RÉSUMÉ
La coque d'arachide, réhydratée à 300 p. 100 en eau salée à 7 p. 1000 , dans une enceinte exposée $48 \mathrm{~h}$ au soleil, permet le développement d'une abondante microflore. Le filtrat, sédimenté, de ce mélange renferme de 24 à 38 p. 100 de matières protéiques brutes, et un mélange d'acides aminés essentiels dont l'indice d'OSER est de 77,1.

Ce sédiment, desséché, ne s'est pas montré toxique in vivo pour le rat ou les volailles.

Incorporé à des taux varıant de 20 à 33 p. 100 à la ration de ces volailles, il réduit leur consommation, donc leur gain de poids, mais leur conserve l'indice de consommation observé avec une ration contenant du tourteau d'arachide.

Le coût de production de ces protéines, avec les méthodes utilisées, est inférieur à ceux des autres procédés actuellement employés en matières de protéines d'organismes unicellulaires (P. O. U.).
\end{abstract}

\section{INTRODUCTION}

La production de protéines d'organismes unicellulaires (P. O. U.-Single cell protein) à partir de divers substrats fait l'objet, depuis quelques années, d'une recherche très active et les travaux ont donné lieu à des revues complètes $(2,6,9)$ et à un Symposium international (3) dont le compte rendu fait le point sur les connalssances actuelles en ce domaine.

Ces recherches s'effectuent encore essentiellement sur la production des P. O. U. à partir de substrats carbonés simples (hydrocarbures, alcanes, paraffines, alcools, sucres, amidon hydro-

(*) Adresse actuelle : C. E. R. Domaine de Pixérécourt, B. P. 9, 54220 Malzéville.

(**) Laboratoire National de l'Elevage et de Recherches Vétérinaires, B. P. 2057, Dakar, République du Sénégal.

(***) Institut d'Elevage et de Médecine Vétérinaire des Pays tropicaux, 10, rue Pierre-Curie, 94700 MaisonsAlfort. lysé) additionnés d'une source d'azote organique ou minéral $(5,7,8)$. Mais très rares sont encore celles qui ont trait à la production des protéines à partir de substrats cellulosiques, réputés difficiles à utiliser par les bactéries, et sans addition d'azote $(1,9)$. C'est l'objet de notre étude, qui emploie par ailleurs des techniques différentes de celles généralement utilisées (fermentation non continue, microflore hétérogène, récolte par décantation) et susceptibles d'abaisser considérablement les coûts de production.

\section{MATÉRIEL ET MÉTHODES}

\section{Matériel}

Substrat: Le substrat est composé de coque d'arachide, résidu du traitement industriel de l'arachide au Sénégal. Cette coque est constituée essentiellement par l'enveloppe ligno-cellulo- 
sique de la graine et parfois quelques résidus de la graine elle-même («son d'arachide») riches en azote.

L'analyse bromatologique de cette coque (*) est donnée au tableau $\mathrm{n}^{\mathbf{0}} 1$, ainsi que celle du produit résultant de sa fermentation microbienne.

Fermenteur : Le fermenteur utilisé est du type silo (fermentation discontinue) à paroi métallique ou bétonnée. L'ouverture supérieure doit être assez large pour permettre un chargement rapide de quantités importantes de coque, mais doit pouvoir être fermée (avec soupape d'échappement des gaz). La phase liquide du produit fermenté doit pouvoir être soutirée par la partie inférieure du silo, après pressage de la phase solide fermentée qui est elle-même évacuée par l'ouverture supérieure.

Microbes utilisés : Contrairement à la plupart des procédés actuels de production de $\mathbf{P}$. O. U., nous n'utilisons pas de souche bactérienne pure, évitant ainsi d'avoir à stériliser préalablement le substrat. Après de nombreux essais (faisant surtout appel à des bactéries du rumen), c'est la microflore naturellement présente au sein de la coque d'arachide qui nous a donné les meilleurs résultats. Cette flore s'est probablement développée et sélectionnée elle-même au cours du stockage des graines d'arachide. Elle présente les caractéristiques générales suivantes, très stables d'un échantillon à l'autre :

- Nombre total des bactéries (après $48 \mathrm{~h}$ d'incubation à $37^{\circ}$ du mélange : coque 25 , eau $75, \mathrm{ClNa} 0,7): 10^{7} \times 9 \pm 2,1$ bact. $/ \mathrm{ml}$.

- Composition de la flore bactérienne : Cocci : 26 p. $100 \pm 7,2$ p. 100 . Bacilles : 72 p. $100 \pm 6,9$ p. 100 . Autres : 2 p. $100 \pm 0,8$ p. 100. Bactéries à coloration «Gram négative»: 62 p. $100 \pm 6,3$ p. 100 . Bactéries à coloration "Gram positive " : 38 p. $100 \pm 5,7$ p. 100.

- Principaux métabolites apparus au cours de la fermentation: Ammoniac : $52,55 \mathrm{mg} / 1 \pm 12,3$. Acide acétique : $0,325 \mathrm{~g} / 1+0,12$. Acide propionique : $0,085 \pm 0,003$. Acide butyrique : $0,205 \pm 0,012$.

$\left(^{*}\right)$ Cette coque est en partie brûlée pour fournir de l'énergie à l'huilerie. Le reste $(50$ à 80 p. 100) est jeté, ou parfois récupéré pour l'alimentation des ruminants ou l'épandage sur les champs.

\section{Méthodes}

\section{Fermentation}

Les méthodes de fermentations microbiennes industrielles, très variées, font généralement appel à la technique dite de "culture continue », c'est-à-dire qu'apport des substrats et soustraction du produit formé se font sans arrêter le processus de fermentation. Malgré son rendement inférieur, nous avons préféré utiliser la méthode de fermentation discontinue, de type «ensilage», en raison de son moindre coût (installation, fonctionnement, entretien). Cette méthode ne permet donc qu'une cellulolyse partielle, et pourrait être améliorée.

Pour initier la fermentation spontanée de la coque d'arachide, il suffit :

1) de la réhydrater ; cette réhydratation se fera de préférence avec de l'eau contenant 6 à $7 \mathrm{p}$. 1000 de chlorure de sodium (*), ajoutée dans la proportion de 3 parties pour une partie de coque d'arachide ;

2) de l'exposer à une température de 30 à $40^{\circ}$. Cette température peut être obtenue, au Sénégal, par simple exposition au soleil durant les mois de mai à septembre.

\section{Récupération des protéines}

Après $48 \mathrm{~h}$ de fermentation, la coque est pressée, débarrassée de sa phase liquide, et rejetée. Cette phase liquide est alors mise à décanter pendant $48 \mathrm{~h}$, puis le surnageant est éliminé et le culot récupéré. Ce culot, qui se présente sous forme d'une boue marron d'odeur aromatique, est desséché en étuve ventilée à $80^{\circ}$. Après cette dessiccation, il ne contient plus que $10^{4} \times 8 \pm 7,2$ bactéries revivifiables par g. Il s'agit donc d'un mélange des éléments solubles (ou de micro-éléments figurés) présents initialement dans la coque, auxquels sont ajoutés les résidus des corps bactériens qui valorisent les précédents.

\section{RÉSULTATS}

La valeur du produit résultant d'une culture destinée à l'obtention de P. O. U. est actuellement bien définie (3-7) par la détermination

(*) En effet, le rendement de la fermentation mesurée par la quantité d'acides gras volatils formés, est accru de 30 à 40 p. 100 lorsqu'on rajoute du chlorure de sodium. Cette eau salée à 7 p. 1000 peut être remplacée par de l'eau de mer, diluée à 20 p. 1000. 
d'un certain nombre de critères qui sont notamment :

1) le rendement global de la culture (poids du produit final/poids du substrat) ;

2) le taux des différents constituants chimiques ou biochimiques du produit (original et final) ;

3) la composition en acides aminés ;

4) la digestibilité, le coefficient d'efficacité protéique ( $"$ Protein efficiency ratio ») et l'utilisation protéique nette ( Net protein utilisation $")$;

5) la recherche des toxines;

6) la valeur alimentaire pour l'animal.

En ce qui concerne le produit obtenu par les procédés décrits précédemment, ces critères ont été ainsi évalués :

1) Rendement : le rendement d'une production de P. O. U. est défini par la quantité (en $\mathrm{kg}$ ) de matière sèche (biomasse) obtenue, par $\mathrm{kg}$ de substrat employé pour l'obtenir.

Dans nos expériences, la moyenne des quantités de matières sèches obtenues était de $181 \pm 12 \mathrm{~g}$ pour $20 \mathrm{~kg}$ de coque d'arachide, soit un rendement moyen de 0,905 p. 100 .

Ce rendement est proche de celui qui est obtenu avec P. O. U. cultivées sur paraffines (3).

2) Taux des différents constituants, chimiques ou biochimiques, du produit obtenu et du substrat de production.

Pour plus de clarté nous ferons figurer sur le même tableau, l'analyse du substrat (coque d'arachide) et du produit final (tabl. 1):

Remarques

1) Ces chiffres concernent l'analyse d'un produit obtenu au mois de décembre. Les produits obtenus au cœur de la saison chaude (juin à septembre) ont une composition différente. En particulier, la teneur en matières protéiques brutes peut s'élever alors à $38,5 \pm 4 \mathrm{p}$. 100 , chiffre observé également lorsqu'on opère en étuve à $37^{\circ}$.

2) Ces chiffres peuvent être rapprochés de ceux rapportés par W. D. BELLAMY (1) à partir de déchets cellulosiques :

Produit original : 23 à 38 p. 100 de cellulose ; 10 à 18 p. 100 de matières protéiques brutes; 2 à 5 p. 100 de matières minérales totales.

Produit final : 6 à 30 p. 100 do cellulose; 30 à 55 p. 100 de matières protéiques brutes ; 2 à 3 p. 100 de matières minérales totales.

La teneur en matières minérales totales est faible comparée à celle que nous avons obtenue avec la coque d'arachide, très chargée en poussières : un dépoussiérage préalable de cette dernière devrait permettre de ramener cette teneur à des proportions acceptables.

TABL. $\mathrm{K}^{\circ} \mathrm{I}$ - Proportion (p.100 de ratiere sçche) les différents élëments dans la coque d'arachide et dans le culct cessêché obtenu après ferrentation.:

\begin{tabular}{|c|c|c|}
\hline Eléments dosés & $\begin{array}{c}\text { Cocue } d^{1}=\text { rachide } \\
\text { (substrat) }\end{array}$ & $\begin{array}{c}\text { Culot desséché de fermentation } \\
\text { (produit final) }\end{array}$ \\
\hline Matières protéiques brutẹs & 8,11 & 23,96 \\
\hline Cellulose & 40,80 & $1 \dot{i}, 911$ \\
\hline Matières grasses & 2,04 & 10,07 \\
\hline Matières minérales totales & 2,42 & $1 \varepsilon, 69$ \\
\hline Extractif non azoté & $\div 6,6-i$ & 32,38 \\
\hline Insoluble chlorhydrique & $C, 44$ & 4,42 \\
\hline Calcium & 0,15 & 0,27 \\
\hline Phosphore & 0,054 & $0,: 25$ \\
\hline Magnésium & 0,12 & 0,19 \\
\hline Potassium & 0,72 & $1,2 I$ \\
\hline Humiditë & $7,95 \mathrm{p}, 1(\mathrm{~m})$ & $3,73,2100$ \\
\hline
\end{tabular}

"Analyse laboratoire I.E.N.V.T. - 94700 Yaiscns-ilfort (rrance). 
3) Composition en acides aminés : elle figure au tableau II.

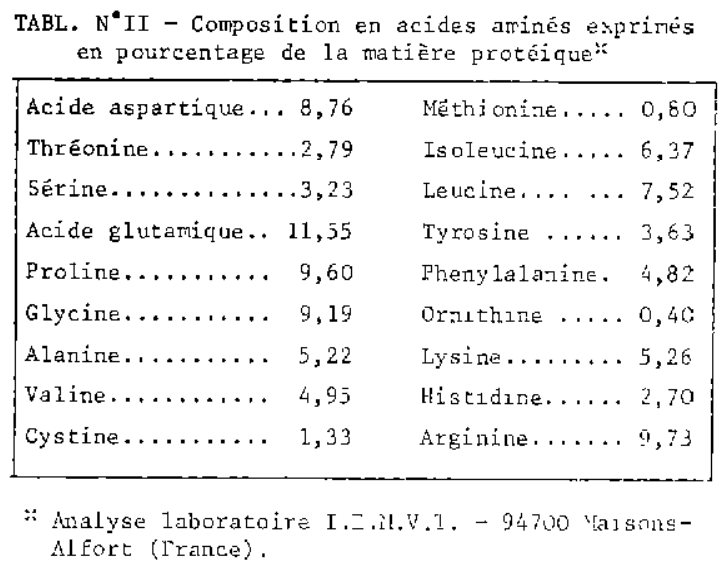

On peut déduire de ce tableau :

\section{1) La classe chimique}

Les acides aminés essentiels (soulignés dans le tableau II) les plus déficients par rapport aux protéines de l'œuf sont de toute évidence, les AA soufrés dont le total (cystine + méthionine) n'atteint que 2,13 p. 100 de la matière protéique.

La classe chimique de la protéine du produit est donc égale à :

Concentration en A. A. soufrés du produit

Concentration en A. A. soufrés de l'œuf

$$
\times 100 \times \frac{2,13}{6,05}=35,2
$$

c'est-à-dire une classe sensiblement égale à celle de nombreux produits végétaux (blé, graines de coton...).

Le pourcentage de déficit en A. A. souftés est de :

$$
\frac{6,05-2,13}{6,05}=64,8 \text { p. } 100
$$

supérieur aux protéines d'oléagineux mais inférieur à celles des graines de légumineuses.

En revanche, le taux de cystine est nettement supérieur à celui des protéines d'oléagineux (soja excepté) et de légumineuses.

\section{2) L'indice d'acides aminés essentiels d'OSER}

77,10 , ce qui correspond à une protéine de valeur moyenne, mais supérieure à la plupart des protéines végétales.
3) La digestibilité, le coefficient d'efficacité protéique et l'utilisation protéique nette $\left({ }^{*}\right)$

Cette recherche est ordinairement effectuée sur rats. Mais compte tenu de la forte teneur en matières minérales et de la teneur relativement élevée en cellulose, cet animal éprouve des difficultés à s'adapter à une ration contenant des taux importants du produit à tester. C'est pourquoi, seul le coefficient de digestibilité apparent de l'azote a pu être déterminé : il est de 17,3 (valeur faible, car il s'agit de protéines bactériennes).

4) Recherches de toxines bactériennes ou de mycotoxines

Cette recherche a été faite in vivo et in vitro.

a) In vivo : des souris, des rats et des poulets ont reçu dans leur ration des taux variant de 10 à 30 p. $100 \mathrm{du}$ produit à tester durant une période minimale de 30 jours. Ni troubles pathologiques, ni mortalités n'ont été observés chez ces animaux durant les essais.

b) In vitro: le produit liquide, résidu de fermentation contient 6 espèces différentes d'Aspergillus, Absidia corymbifera, Mucor sp. et Cladosporium sp. Après dessiccation à $80^{\circ}$, seul ce dernier genre est retrouvé dans le produit (**).

Aucune bactérie pathogène ou toxinogène n'a pu être mise en évidence par les techniques classiques.

\section{5) Valeur alimentaire pour les volailles}

Afin d'apprécier la valeur alimentaire des protéines contenues dans le produit étudié, nous l'avons incorporé, dans des rations destinées à des poussins de 8 jours (lot de 12 individus), en remplacement du tourteau d'arachide.

La croissance pondérale de ces poussins a été comparée durant 30 jours à celle de sujets témoins (lot 2) recevant une ration de composition suivante :

- farine de maïs : 25 p. 100

- farine de sorgho: 25 p. 100

- remoulage de blé : 18 p. 100

- farine de poisson : 9 p. 100

(*) Nous remercions vivement le Docteur M. NDIAYE, Directeur de l'Organisme de Recherches sur l'Alimentation et la Nutrition Africaine à Dakar, qui a bien voulu effectuer ces mesures.

(**) Analyse Laboratoire de Pharmacologie-Toxicologie de 1'I. N. R. A., 31300 Toulouse (France). 
- complément protéique (sous forme de tourteau d'arachide) : 20 p. 100

- carbonate de chaux : 1,2 p. 100

- chlorure de sodium : 1,2 p. 100

- mélange polyvitaminé : 0,6 p. 100

N. B. Ce complément protéique varie selon les lots (voir tableau $\mathrm{n}^{\circ} 3$ ) ; dans la ration des lots 1 et 4 , la proportion des autres composants est ajustée en conséquence, mais les rations ne sont pas isoazotées.

Un premier lot témoin dont la ration ne contient ni tourteau ni produit à tester, a été constitué, afin de vérifier si l'absence de complément protéique n'entraînait pas une croissance au moins égale à celle provoquée par le produit (lot 3).

Les résultats sont les suivants :

TABILEL $\Sigma^{\circ}$ III

\begin{tabular}{|c|c|c|c|c|}
\hline $\mathrm{L} \circ \mathrm{ts}$ & 1 & 2 & 3 & 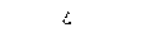 \\
\hline Comp lêment protéique & Néant & $\begin{array}{l}\text { Tourteau } \\
\mathrm{d}^{\prime} \text { arachide } \\
20 \mathrm{p} \cdot 100\end{array}$ & $\begin{array}{l}\text { Prodult i } \\
\text { tester } \\
20 \mathrm{p} \cdot 100\end{array}$ & $\begin{array}{l}\text { Frodut L ì } \\
\text { tester } \\
33 \mathrm{p} \cdot 100\end{array}$ \\
\hline $\begin{array}{l}\text { Teneur en MPB de } \\
\text { la ration }\end{array}$ & $17.75 \mathrm{p} .100$ & $23,50 \mathrm{p} \cdot 100$ & $19,00 \mathrm{p} .100$ & $19.80 \mathrm{p} .100$ \\
\hline $\begin{array}{l}\text { Gain de poicls } \\
\text { royen quotrdien }\end{array}$ & $6,55 \mathrm{~g}$ & $10,66 \mathrm{~g}$ & $8,6 \mathrm{~g}$ & $5,51 \mathrm{~g}$ \\
\hline $\begin{array}{l}\text { Quantité d'aliment } \\
\text { consonmé par jour }\end{array}$ & $19,14 \mathrm{~g}$ & $29,51 \mathrm{~g}$ & $23,96 \mathrm{~g}$ & $76,48 \mathrm{~g}$ \\
\hline $\begin{array}{l}\text { Indice de consonma- } \\
\text { tion (g d'aliment } \\
\text { par g de poics) }\end{array}$ & $2,92 \mathrm{~g}$ & $2,76 \mathrm{~g}$ & $2,78 \mathrm{~g}$ & $2,62 \mathrm{~g}$ \\
\hline
\end{tabular}

L'incorporation du produit dans la ration entraîne donc une diminution de la consommation et du gain de poids quotidien, d'autant plus forte que la proportion est plus grande, mais les indices de consommation des lots 2,3 et 4 ne sont pas significativement différents. Cette sousconsommation tient probablement à l'encombrement relatif plus élevé de la ration, qui freine l'appétit des volailles et a une teneur plus faible en protéines.

L'autopsie des volailles en expérience ne révèle aucune différence par rapport aux témoins. On ne note, non plus, aucune différence quant aux caractères organoleptiques de leur chair.

\section{DISCUSSION}

Les différentes analyses réalisées sur le produit résiduel de la coque d'arachide fermentée permettent, par leur résultat, de la comparer favorablement à la moyenne des protéines unicellulaires bactériennes obtenues par d'autres méthodes. Toutefois, il convient de bien remarquer que le produit analysé est brut, c'est-à-dire contient un mélange de protéines bactériennes et de certaines protéines solubles déjà présentes dans la coque d'arachide.
Les essais préliminaires de valeur alimentaire effectués sur volailles donnent des résultats très proches de ceux obtenus avec d'autres protéines d'origine microbienne, en particulier, celles du microchampignon Fusarium graminareum testées par I. F. DUTHIE (9). Ce dernier auteur notait une baisse de consommation (done de gain de poids) de 12 à 18 p. 100 lorsque le taux d'incorporation de la protéine microbienne atteint 30 p. 100 ; cette diminution s'annule lorsqu'on réduit le taux d'incorporation à 10 p. 100 , pour un indice de consommation (= «Feed conversion ratio») égal à celui des témoins.

Par analogie, il conviendrait donc, dans les cas des protéines obtenues sur coque d'arachide fermentée :

1) D'accroître la teneur en matières protéiques brutes (on peut atteindre 35 p. 100 en effectuant les fermentations durant la saison chaude) ;

2) De réduire le taux d'incorporation à $10 \mathrm{p}$. 100.

Dans ces conditions, et compte tenu du très faible coût de la protéine (de 60 à 80 p. 100 inférieur à celui du tourteau), l'utilisation du produit de fermentation de la coque d'arachide pourrait s'avérer rentable. 


\section{CONCLUSION}

La fermentation spontanée de la coque d'arachide, réhydratée par de l'eau salée et exposée à la chaleur tropicale, permet la multiplication d'une abondante flore bactérienne. Une partie de cette flore, recueillie par simple décantation avec des résidus du substrat, peut être considérée comme une source éventuelle intéressante de protéines d'organismes unicellulaires.
Les résultats des différentes analyses chimiques, biochimiques et toxicologiques ainsi que ceux des essais in vivo sur animaux, permettent de penser que ces protéines pourraient être incorporées à des aliments destinés aux volailles. L'intérêt majeur de la production de telles protéines reste leur prix de revient faible, le matériel de production étant très sommaire et le substrat utilisé ayant une valeur commerciale nulle.

\section{SUMMARY}

\section{Single cell protein production from peanut hulls}

When peanut hulls rehydrated (300 p. 100$)$ in salt water ( 7 p. 1000$)$ are left in a tank during 48 hours under tropical sun an important bacterial cells growth occurs. The bulk of this mixture, after filtration, contains 24 to 38 p. 100 of protein with an amino-acids OSER index of 77.1.

Dried, this product proved atoxic for rats and poultry.

When incorporated in chicken feed, at 20 to 33 p. 100 levels, average feed intake and body weight gains are reduced, but feed conversion ratio is still the same that in controls fed with equivalent levels of groundnut cake.

Production costs, with described methods, are lower than others usual single cells protein processings.

\section{RESUMEN}

\section{Producción de proteinas unicelulares a partir de cáscaras de cacahuete}

La cáscara de cacahuete, rehidratada a 300 p. 100 con agua salada a 7 p. 1000 , en un recinto orientado al sol durante 48 horas, permite el desarrollo de una microflora abundante.

En el líquido filtrado, sedimentado, de esta mezcla se hallan 24 a 38 p. 100 de materias proteicas y una mezcla de amino-ácidos esenciales cuyo índice de Oser es de 72,1.

Este sedimento, desecado, no se mostró toxico in vivo para la rata o las aves de corral.

Tasas de 20 a 33 p. 100 de dicho sedimento incorporadas en la ración de estas aves reducen su consumo, pués su aumento de peso, pero conservan el índice de consumo observado con una ración conteniendo torta de cacahuete.

El coste de producción de estas proteinas, con los métodos utulizados, es inferior al de los otros procedimientos actualmente empleados tratandose de proteinas unicelulares.

\section{BIBLIOGRAPHIE}

1. BELLAMY (W. D.). Single cell proteins from cellulosic wastes. Biotech. Bioeng. 1974, 16 (7): 869-880.

2. BOMBAL (J.), NDIAYE (L.), FENARDJI (F.), FERRANDO (R.). Concurrence des protéines conventionnelles et des protéines non conventionnelles. Rev. Méd. vét., 1974, 125 (4) : 469-491.

3. DAVIS (P.). Single cell protein. Proceedings of the international Symposium Rome, 1973. London, N. Y., S. F., Academic Press, 1974.

4. DREYFUS (A.). Etude de la production de protéines d'organismes unicellulaires à partir du manioc. DEA de microbiologie. I. R. C. H. A. 91710 Vert-Le-Petit (France), 1975.
5. FERRANDO (R.). Au sujet des protéines unicellulaires et de leur nomenclature Annls Hyg. L. Fr. Méd. Nut. 1974 (4) : 347-349.

6. JACQUET (J.). Sur le domaine de la microbiologie alimentaire. C. R. Acad. Agric. 1967 (7) : 503-514.

7. LICHTFIELD (J. H.). The facts about food from unconventional sources. Chem. Proc. 1974, 20 (9) : 1118.

8. SENEZ (J. C.). Acquisitions et perspectives économiques de la microbiologie du pétrole. Bull. Inst. Pasteur, 1969,67 (8) : 1771-88.

9. TANNEMBAUM (S. R.), WANG (D. I. C.). Single cell protein II. Cambridge, London, M. I. T. Press, 1975 . 